Supplementary Information for

\title{
Functional dynamics of an ancient membrane-bound hydrogenase
}

Max E. Mühlbauer ${ }^{1,2}$, Ana P. Gamiz-Hernandez¹, Ville R. I. Kaila1,2*

${ }^{1}$ Department of Biochemistry and Biophysics, Stockholm University, 10691, Stockholm, Sweden.

2 Department of Chemistry, Technical University of Munich, Lichtenbergstraße 4, 85748 Garching, Germany.

* Corresponding author: Ville R. I. Kaila

Email: ville.kaila@dbb.su.se

This PDF file includes:

SI Methods

Figures S1 to S10

Tables S1 to S5

Movie S1

SI References 


\section{SI Methods}

\section{Definition of water pathways}

Based on clustering analysis (see main text Methods), we define pathways/wires/channels (used here interchangeably) as follows: A pathway connects an area on the protein surface ('inlet') to a site of interest ('object'). The trajectories of all water molecules coming close to the object at any timepoint during simulations, are clustered according to the inlet along which the waters enter the protein interior. Only parts of the trajectories inside the protein (approximated by a convex hull) are considered for the analysis. A pathway is defined here as the medoid of each cluster of pathways, i.e., an inlet cluster.

\section{Structural perturbation of TM3}

TM3 of MbhD was modeled in an $\alpha$-helical form by adding harmonic restraints with a force constant of $400 \mathrm{kcal} \mathrm{mol}^{-1}$ per residue on the $\alpha$-helical secondary structure content of residues 63 to 88 using a collective variable, as implemented in the colvar module of NAMD2. Backbone hydrogen bonds were additionally restrained for residues 71 to 79 with a harmonic force constant of $50 \mathrm{kcal} \mathrm{mol}^{-1} \AA^{-2}$ on the distance between the $\mathrm{N}$ atom of residue $i$ and the $\mathrm{O}$ atom of residue $i+4$.

\section{Poisson-Boltzmann electrostatic calculations with Monte Carlo sampling}

Initial protonation states for the MD simulations were derived based on Poisson-Boltzmann electrostatic calculations with Monte Carlo sampling of different protonation states (PBE/MC). To this end, a minimized cryoEM structure setup of Mbh with missing regions modeled in silico (see Methods) was prepared. For the PBE/MC calculations, the protein was treated as atomic point charges, and embedded in a polarizable dielectric continuum with a dielectric constant of $\varepsilon=4$. The bulk and buried water cavities were modeled as dielectric cavity with $\varepsilon=80$. $p K_{a}$ values were estimated based on electrostatic interactions, the desolvation energy of transferring the titratable groups in protonated and deprotonated states from bulk to the protein interior, and by sampling the $2^{N}$ protonation states of the titratable residues using the $\mathrm{MC}$ method with single, double, and triple moves in the protonation state space. The PBE/MC calculations were performed by coupling the Adaptive-Poisson Boltzmann Solver ${ }^{1}$ and CHARMMv. $38 .{ }^{2}$ The Monte Carlo-sampling was performed using Karlsberg+ ${ }^{3,4}$

\section{DFT calculations}

Reaction pathway optimizations for the proton transfer within the NiFe center were performed in DFT models based on geometry optimized states with the proton on Glu21L, Cys374L, or in the $\mathrm{H}_{2}$-bound states receptively using a zero-temperature string-type of method, ${ }^{5}$ followed by optimization of the transition state. The initial reaction pathway was constructed by interpolating between optimized proton donor and acceptor states in 21 intermediate structures via the linear synchronous transit method. The reaction pathway was further optimized with equally spaced intermediate structures, constrained by a quadratic potential until the RMSD of the gradient varied $<10^{-4}$ au, followed by optimization of transition states. The reaction energetics was benchmarked at the B3LYP-D3, ${ }^{6}$ B3LYP ${ }^{*},{ }^{7}$ CAM-B3LYP,${ }^{8}$ CAMhB3LYP, ${ }^{9}$ and $\omega B$ B $97 X-D,{ }^{10}$ levels with def2-TZVP basis sets on all atoms. 

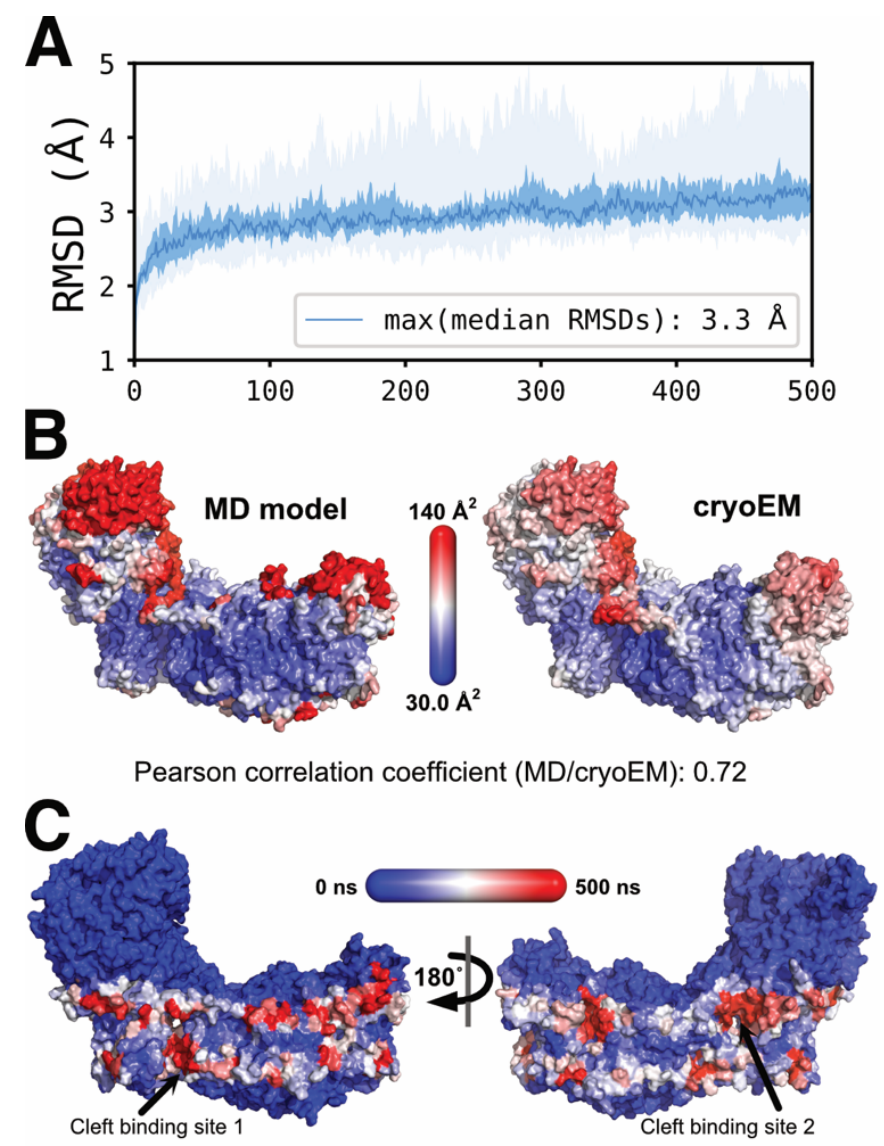

Figure S1. (A) Root mean square deviation (RMSD) of Mbh during the simulations. The dark blue line corresponds to the median RMSD across all 16 simulations. Medium blue shaded area indicates the region between the 25 percentile and the 75 percentile and light blue area the area between the minimum and maximum value for each timepoint. (B) B-factors calculated from MD simulations (left) and experimental B-factors extracted from the cryoEM map. (C) The residue-wise lipid residency time (from blue, $0 \mathrm{~ns}$, to red, $500 \mathrm{~ns}$ ) shows two highly stable lipid binding sites in the putative binding cleft between $\mathrm{MbhH}$ and $\mathrm{MbhM}$. The calculation was performed using pyLipid. ${ }^{11}$ 

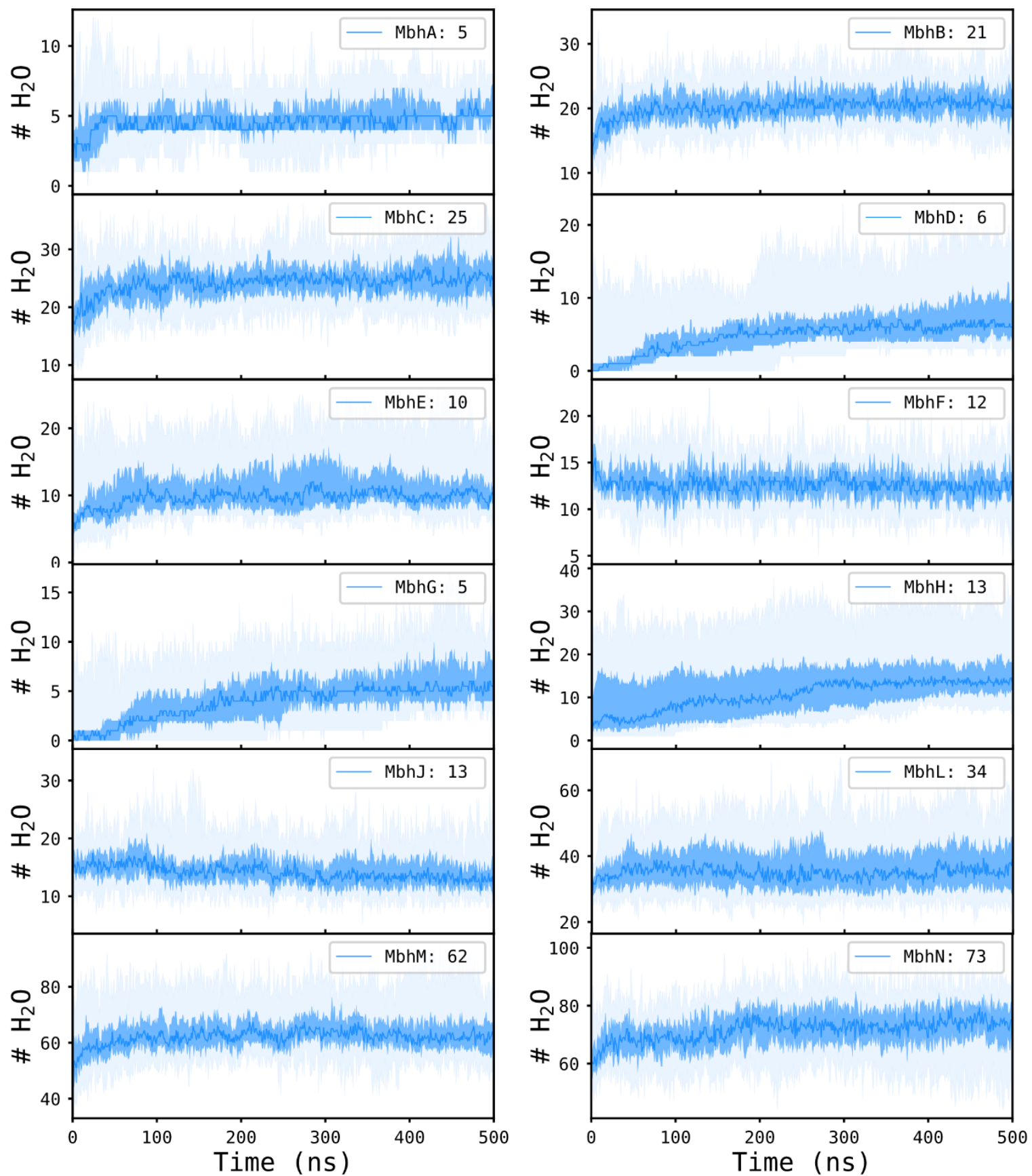

Figure S2. Water counts around conserved functional residues in subunits of Mbh (see Table S4). Blue lines indicate the median over all simulations, blue shaded area the extent between the 25 and 75 percentile and light blue shaded area the observed minimum/maximum values in all simulations. 

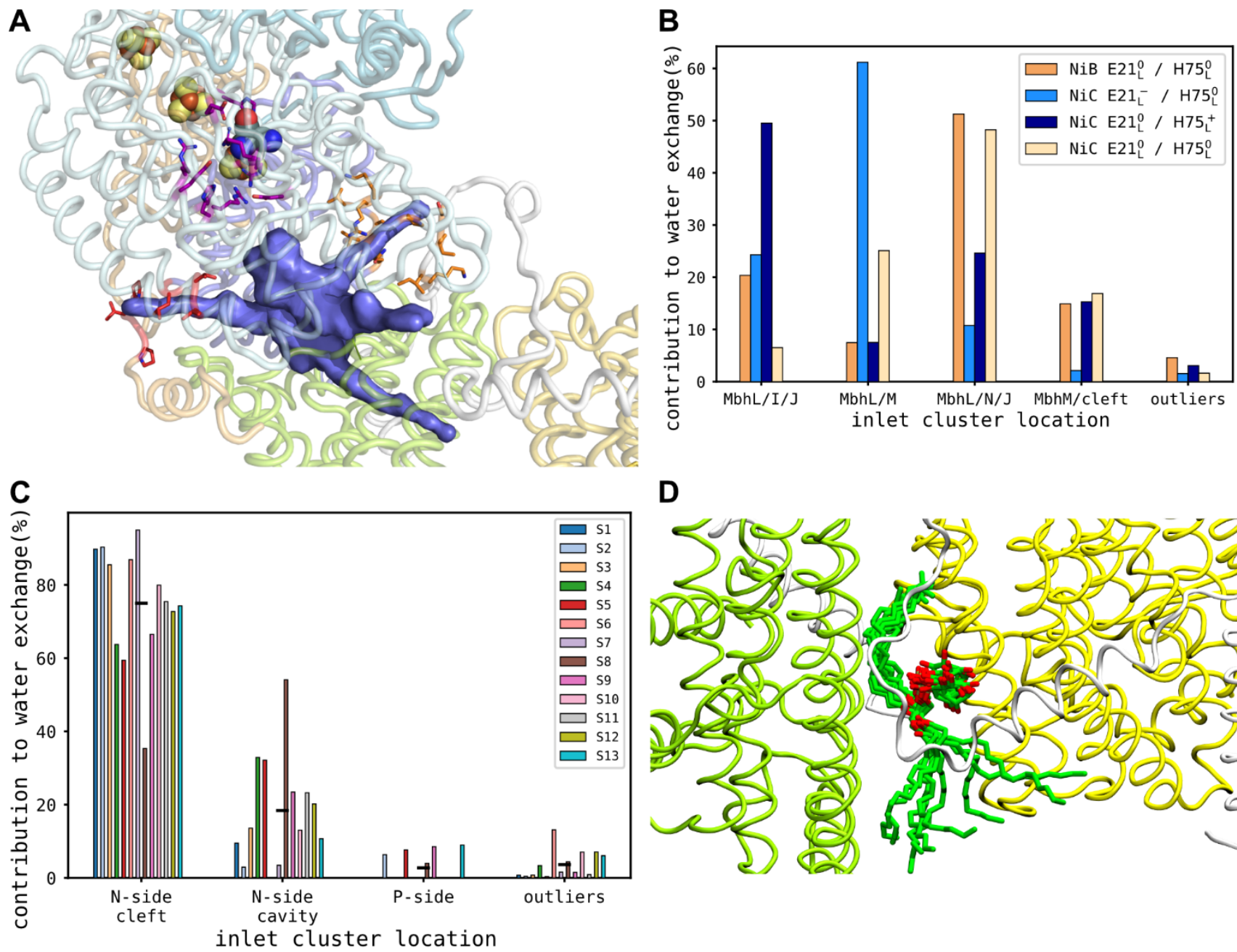

D

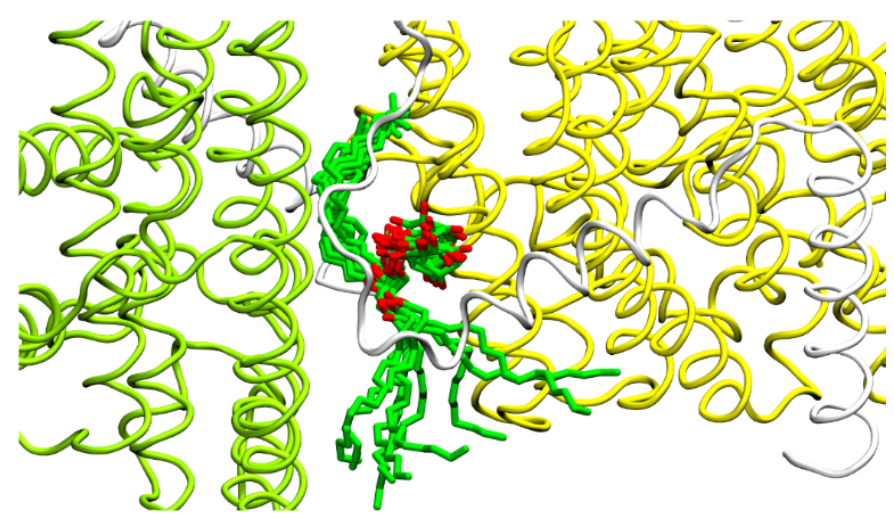

Figure S3. (A) Comparison of the four putative proton pathways leading to Glu21 (cluster centroid paths shown as blue surface) and comparison to previously identified pathways indicated by their tunnel lining residues shown as sticks. Residues along the pathway described by Grba et al. ${ }^{12}$ for the NiFehydrogenase from Desulfovibrio vulgaris leading to E21 L via His75 (magenta), and pathways leading to the Q-cavity of complex I based on previous simulations (orange and red). ${ }^{13} \mathrm{His} 75 \mathrm{~L}$, which could serve as a second/alternative donor of a proton to the NiFe site, was modeled both in its protonated and deprotonated states (see Methods). (B) Contributions of water clusters leading to the active site of $\mathrm{MbhH}$ in different states of the NiFe-cluster and surrounding residues. (C) Contributions of water clusters leading to the hydrophilic axis of $\mathrm{MbhH}$ in the individual simulation. The black horizontal lines indicate the median over all simulations. (D) Most commonly observed binding poses of the additional lipid molecules that could fit to the cleft from the $\mathrm{N}$-side based on a pyLipid analysis ${ }^{11}$ of simulation $\mathrm{S} 6$. 

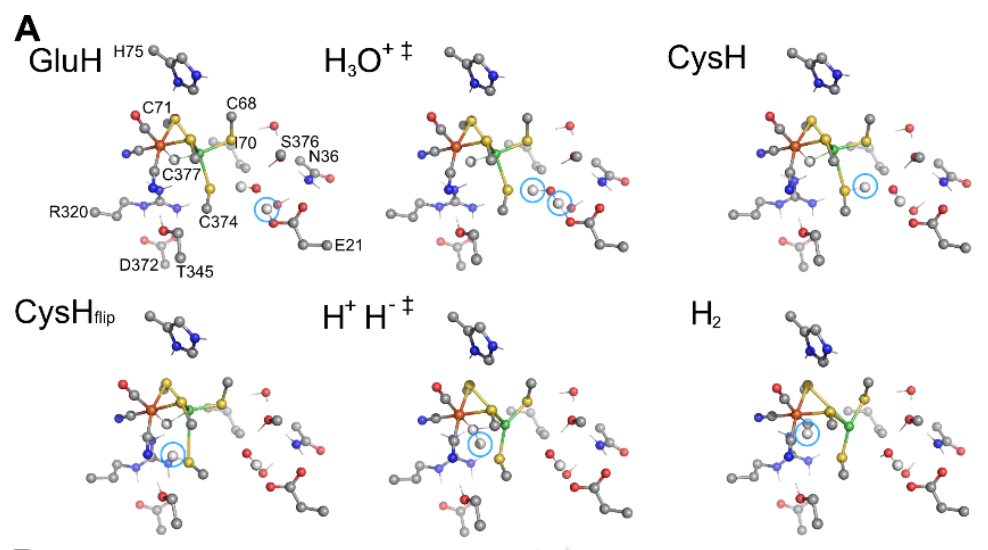

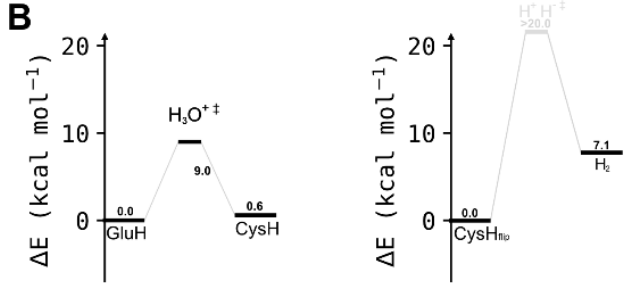

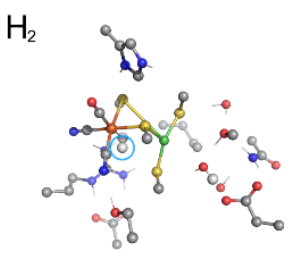

Figure S4. (A) Optimized DFT models of the different states along the catalytic cycle. (B) Relative electronic energies for pT from Glu21 to Cys374 (left) and Cys374 to $\mathrm{H}_{2}$ (right) in the Fe"Nill $\mathrm{S}=1$ state, calculated at the B3LYP-D3/def2-TZVP/e=4 level. See also Methods and Table S5 for energetics calculated with different density functionals. 
A

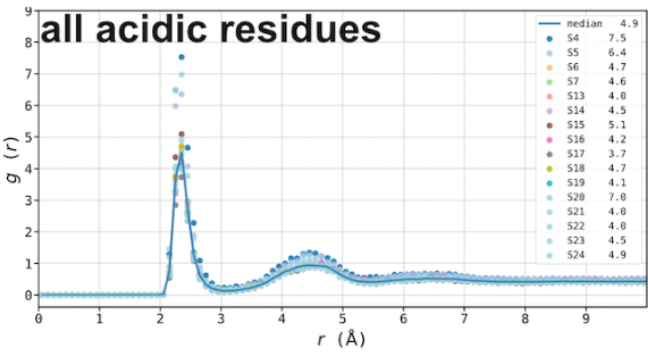

C

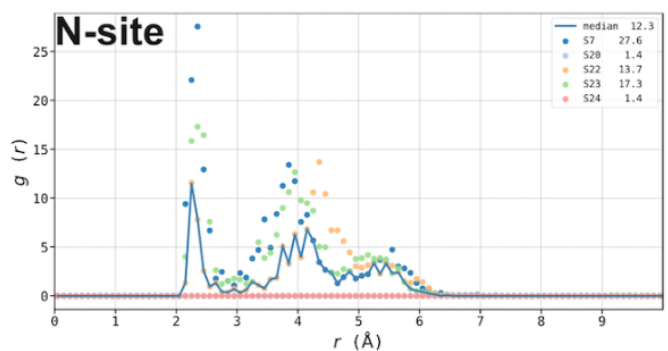

E

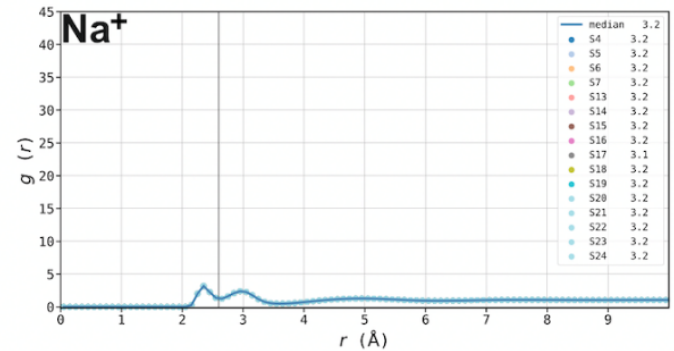

G

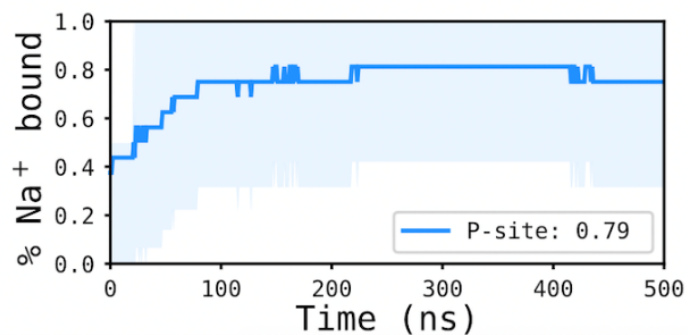

B

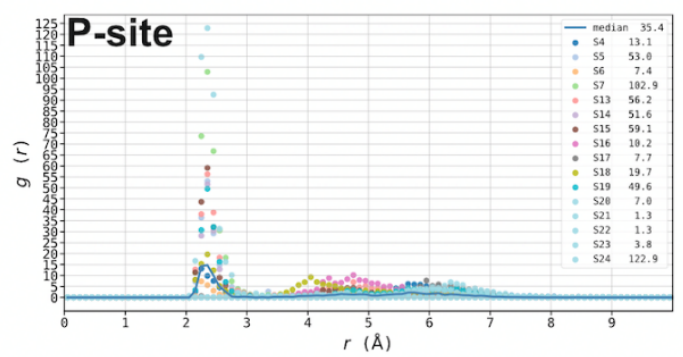

D

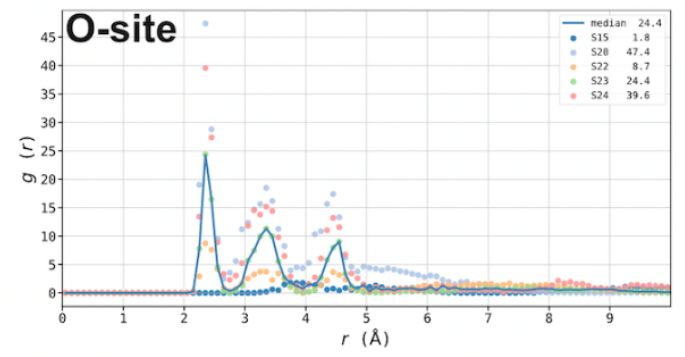

$\mathbf{F}$

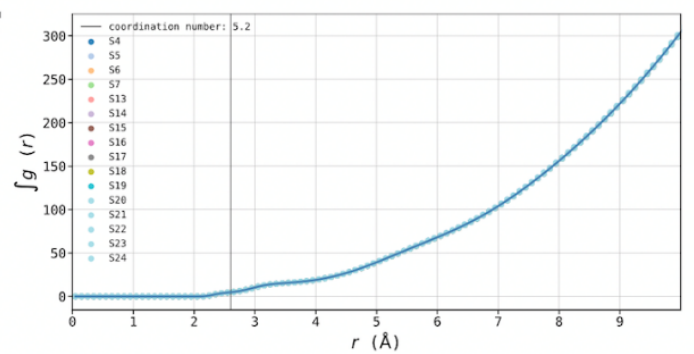

Figure S5. Radial distribution functions $(g(r))$ of sodium ions around residues in the putative binding sites. (A) The $g(r)$ for $\mathrm{Na}^{+}$around all acidic residues in Mbh shows a 4-fold enhanced maximum probability density around general acidic residues. $g(r)$ for $\mathrm{Na}^{+}$based on $\mathrm{MD}$ simulations from (B) Pside His59 ${ }_{\mathrm{B}}$ (30-fold enhancement), (C) N-site Asp35 (12-fold enhancement), and (D) O-site His41 (25-fold enhancement). (E) $g(r)$ around sodium ions indicates that the first solvation shell around the cations is reproduced correctly in our simulations. (F) Integration of $g(r)$ around sodium ions up to the first minimum yields a coordination number of 5.2 in good agreement with previous studies. ${ }^{14}$ The first minimum is indicated by vertical black lines in $\mathrm{E}$ and $\mathrm{F}$. (G) \%- of simulation time that $\mathrm{Na}^{+}$binds to the $\mathrm{P}$-site. The blue line shows the fraction of simulations, which have a sodium ion bound at the P-site at a given timepoint, with the shaded area indicating the standard deviation. On average, ca. $80 \%$ of the simulations have one sodium bound at the P-site. 

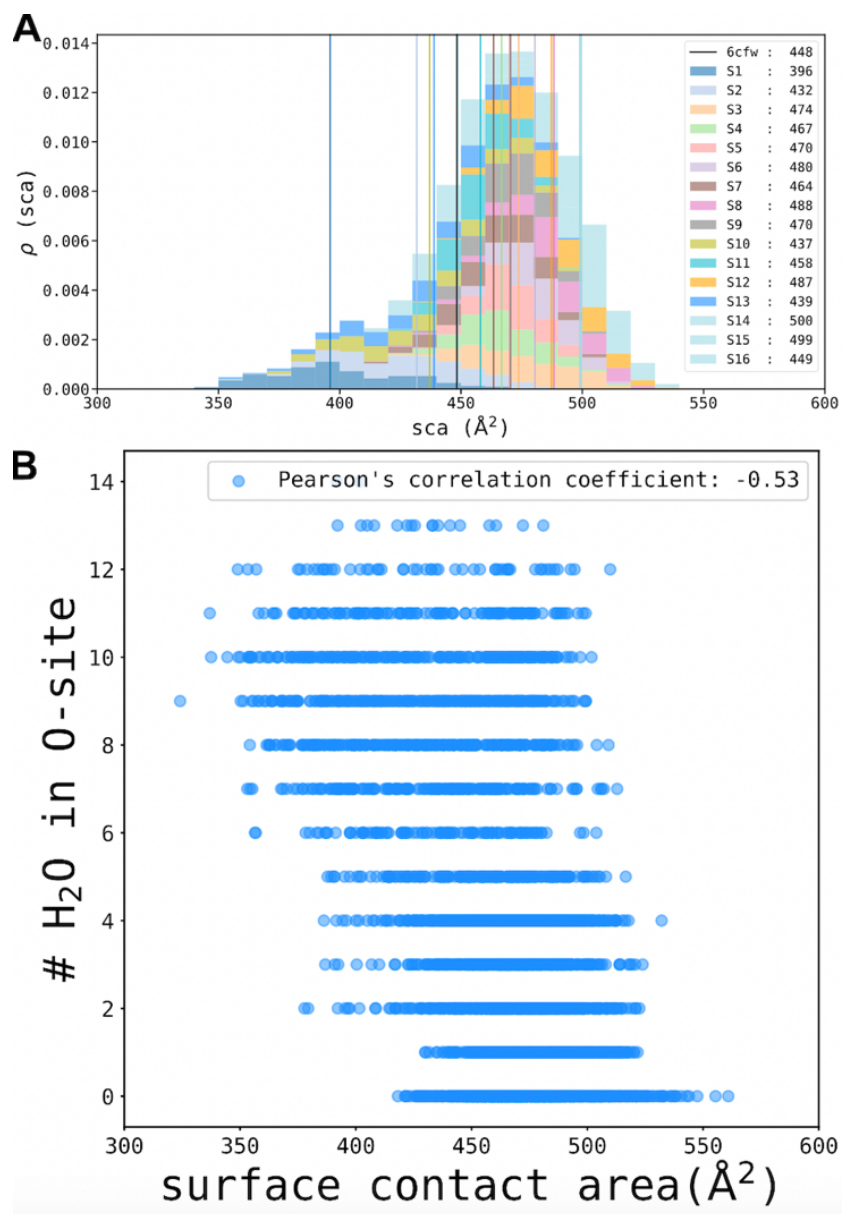

Figure S6. Surface contact area (SCA) and hydration in MbhD. (A) SCA between TM2 (residue 13-60) and TM3 (residue 63-87) of subunit MbhD in different MD simulations. Legend labels and horizontal bars indicate median SCA from individual simulations and the SCA computed from the cryoEM structure (PDB ID: 6cfw), respectively. (B) Anti-correlation between the hydration of the O-site and the SCA supports its possible gating function. Hydration and sca were measured for all simulations without sodium ion in the $\mathrm{N}$ - or O-sites. 

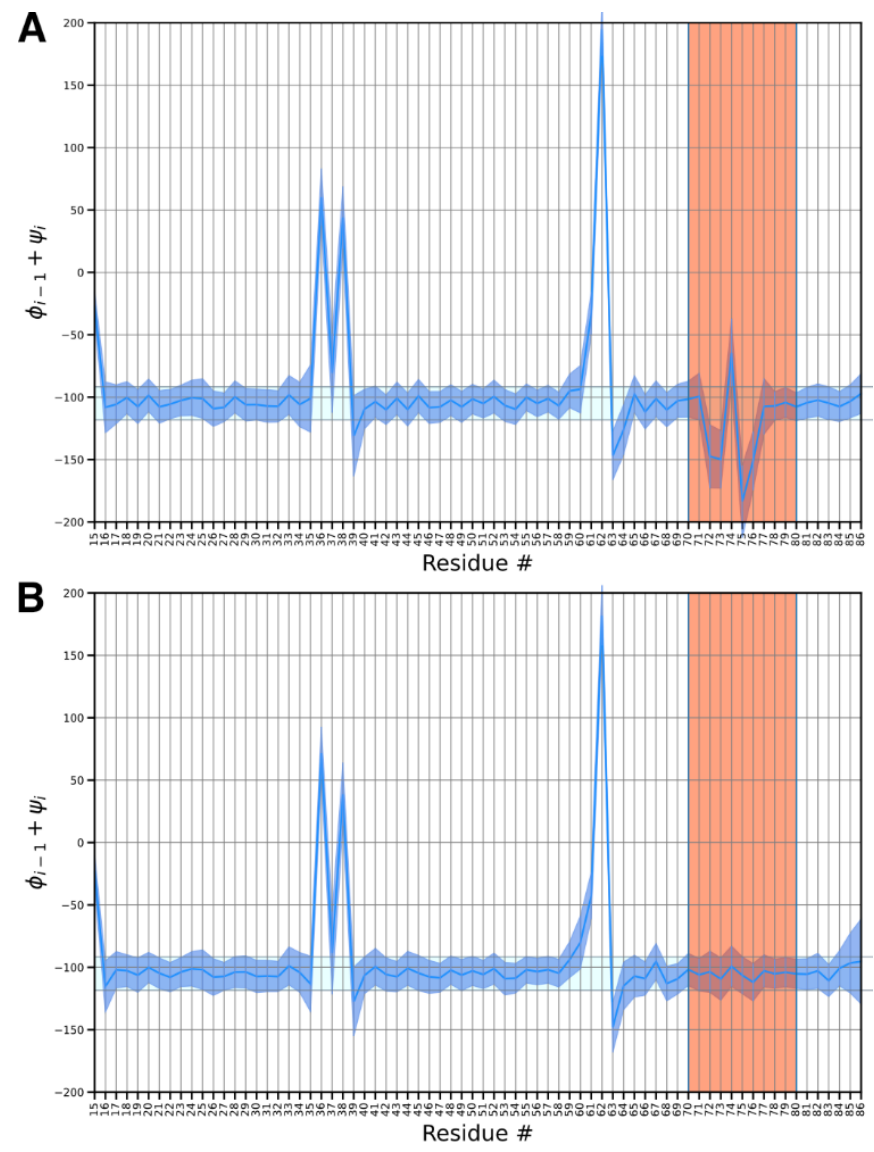

Figure S7. Sum of backbone dihedral angles $\left(\varphi_{i-1}+\psi_{i}\right)$ as a measure for the $\alpha$-helicality of residues in subunit MbhD. Backbone angles of ca. $105^{\circ}$ indicate an $\alpha$-helix, while deviations from this value indicate the $\pi$-bulge. The blue trace represents a median over the whole simulation for each residue, and the blue shaded area shows 25/75-percentiles. (A) Unbiased MD simulation (S5), which shows a strong deviation from an ideal $\alpha$-helix (shared area) (residues 70-80). (B) Simulation S13 was biased to form an ideal $\alpha$-helix, which remains stabled during the complete (500 ns) simulation timescale. 

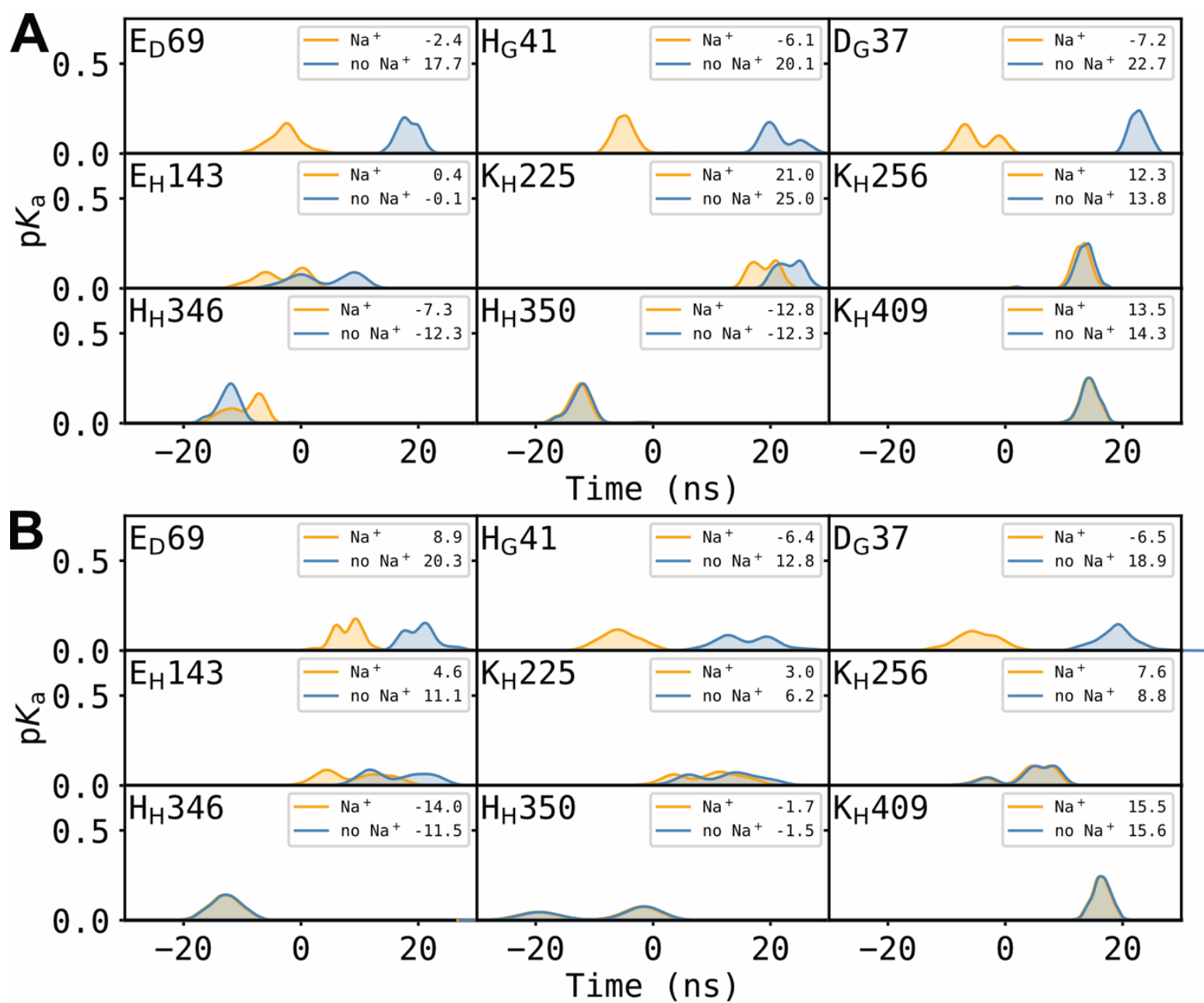

Figure S8. The influence of $\mathrm{Na}^{+}$binding on the transient $\mathrm{p} K_{\mathrm{a}}$ of titratable residues in the $\mathrm{MbhD} / \mathrm{G} / \mathrm{H}$ subunits based on electrostatic calculations. (A) $p K_{\mathrm{a}}$ distributions from simulation S12 (closed Glu143 $\mathrm{H}^{-}$ Lys225 $\mathrm{H}$ ion-pair) and (B) S16 (open Glu143H-Lys225 $\mathrm{H}$ ion-pair). Sodium binding lowers the proton affinity from Glu69D/His41G/Asp37G cluster, which lead to protonation of Glu143H. 

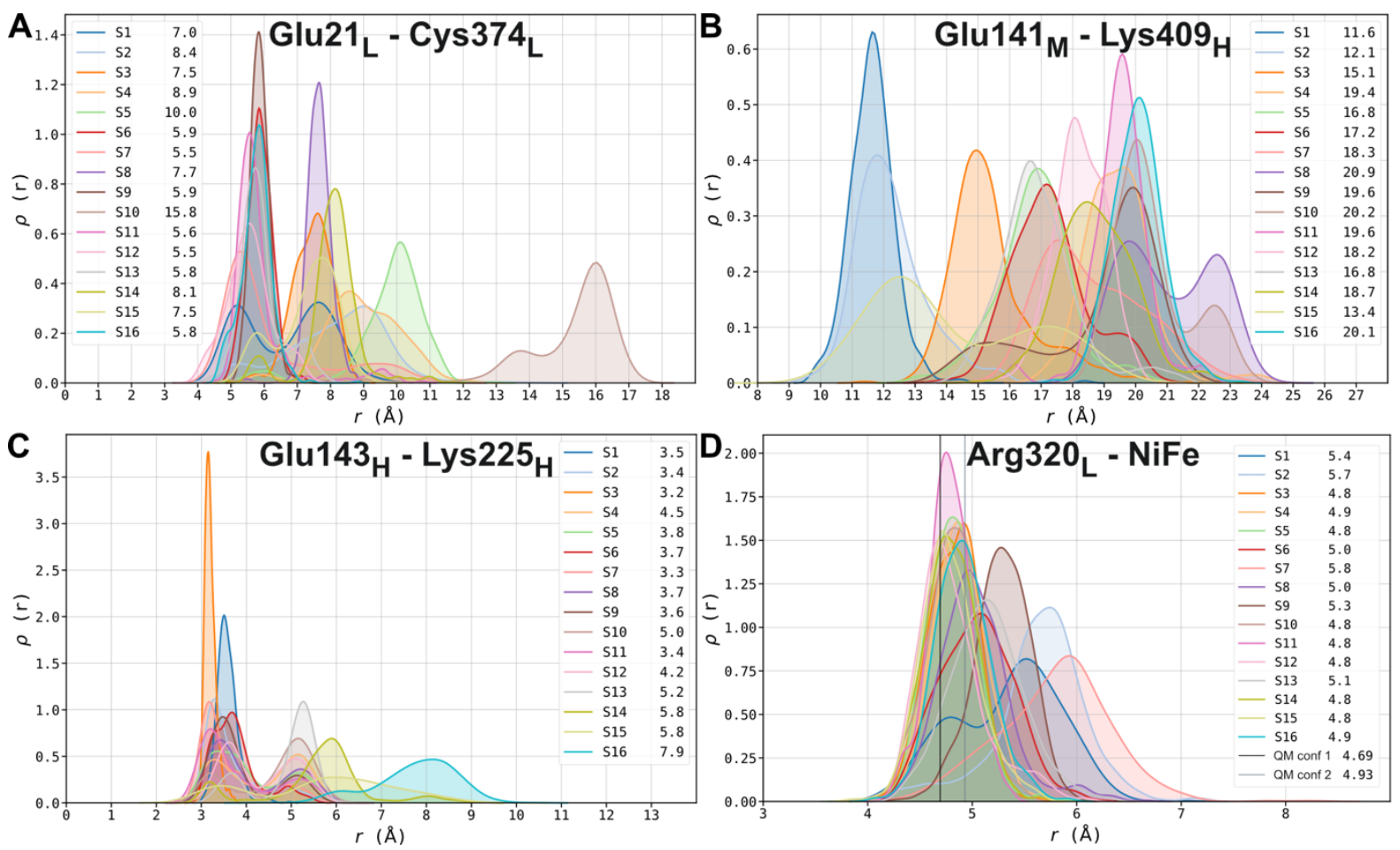

Figure S9. Distance distributions during MD simulations. (A) Distance distribution between Glu21L $(\mathrm{C} \delta)$ and $\mathrm{Cys} 374 \mathrm{~L}(\mathrm{~S} \gamma)$. Glu21 $\mathrm{L}$ was modelled in a protonated state in all simulations except in simulation S10 (pink), where the residue flips away from the active site. Labels report median distances. (B) Distribution of distances between Glu141 $\mathrm{M}(\mathrm{C} \delta$ ) and Lys409M $(\mathrm{Nz})$. Glu141M was modelled in a deprotonated state in simulations S1 and S2 (blue/light blue), and protonated in the other states, which increases the distance between the residues. (C) Distances distribution of between Glu143 $\mathrm{H}(\mathrm{C} \delta$ ) and Lys225 $5_{\mathrm{MH}}(\mathrm{Nz})$, and (D) between Arg320 $(\mathrm{Cz})$ and the NiFe (center of mass). 

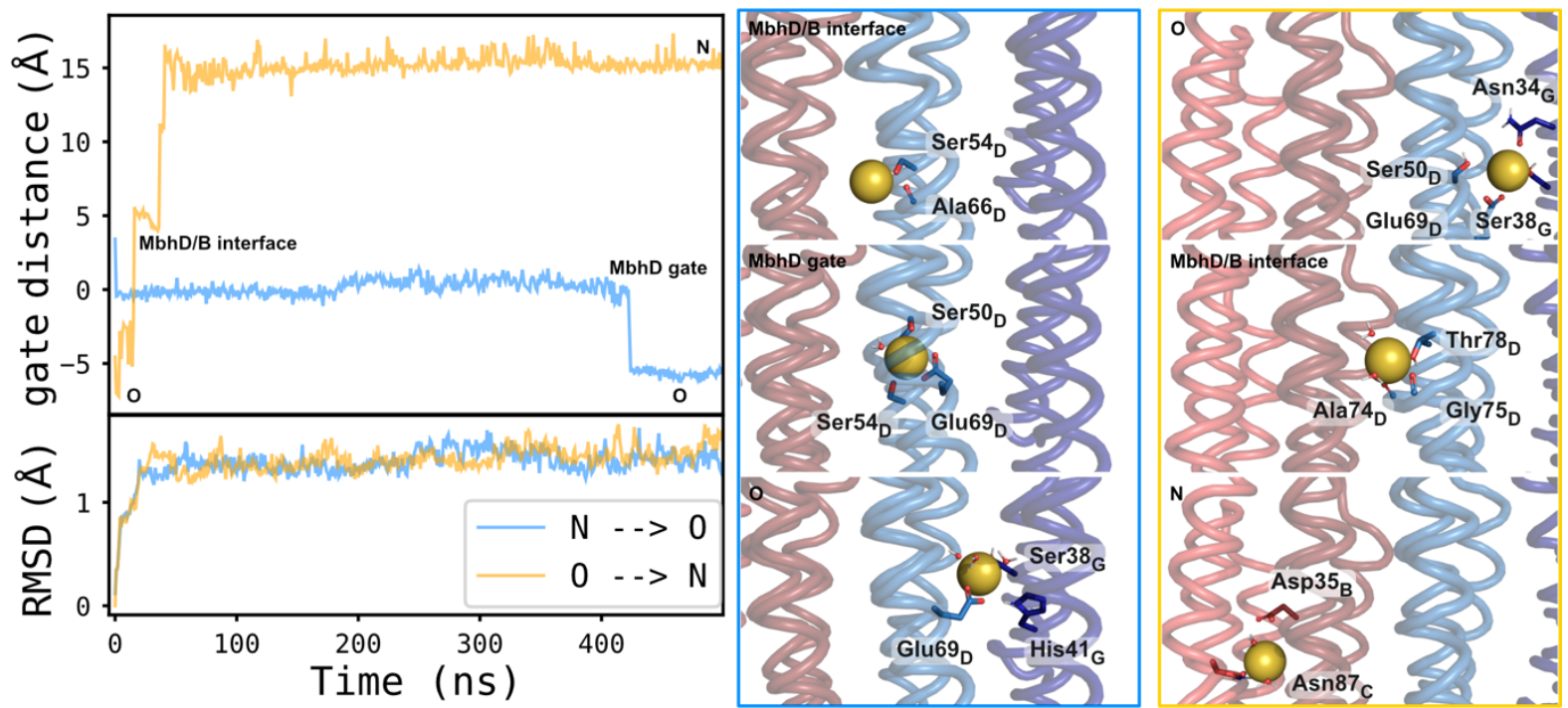

Figure S10. Sodium dynamics in the MbhD gate. $\mathrm{Na}^{+}$passing through the $\mathrm{MbhD}$ gate in $\mathrm{O} \rightarrow \mathrm{N}$ site direction (blue) and the $\mathrm{N} \rightarrow \mathrm{O}$ site direction (yellow). (A) The gate distance (top) shown as the perpendicular distance between the sodium and a plane fitted through the $\mathrm{C} \alpha$-atoms of MbhD. The RMSD of MbhD (bottom) during the $\mathrm{O} \rightarrow \mathrm{N}$ and $\mathrm{N} \rightarrow \mathrm{O}$ simulations. The residues forming stable interactions with the $\mathrm{Na}^{+}$during the $500 \mathrm{~ns} \mathrm{MD}$ trajectories are shown to the right. 
Table S1. Summary of performed Mbh simulations. Abbreviations: PYPI - 1-palmitoyl-2-palmitoyl-

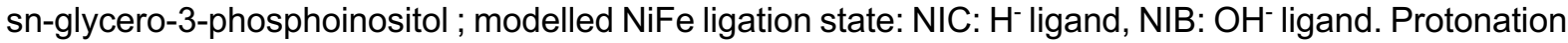
states: std - standard; PBE - based on electrostatic calculations (see Table S2).

\begin{tabular}{|c|c|c|c|c|c|c|}
\hline Simulation & $\begin{array}{l}\text { Starting } \\
\text { point }\end{array}$ & Non-standard setup & Cofactor & Lipid & Protonation & Length [ns] \\
\hline $\mathrm{S1}$ & $6 \mathrm{cfw}$ & - & NIB & PYPI & std & 500 \\
\hline S2 & $6 \mathrm{cfw}$ & - & $\mathrm{NIC}$ & PYPI & std & 500 \\
\hline S3 & $6 \mathrm{cfw}$ & - & $\mathrm{NIC}$ & PYPI & PBE & 500 \\
\hline S4 & $6 c f w$ & Na placed in N-site & $\mathrm{NIC}$ & PYPI & PBE & 500 \\
\hline S5 & $6 \mathrm{cfw}$ & - & $\mathrm{NIB}$ & PYPI & PBE & 500 \\
\hline S6 & $6 \mathrm{cfw}$ & N-leaflet lipid in cleft & NIB & PYPI & PBE & 500 \\
\hline S7 & $6 \mathrm{cfw}$ & Na placed in I-site & $\mathrm{NIC}$ & PYPI & PBE & 500 \\
\hline S8 & $6 \mathrm{cfw}$ & $\mathrm{H} 232^{+} \mathrm{H}$ & $\mathrm{NIC}$ & PYPI & PBE & 500 \\
\hline S9 & $6 \mathrm{cfw}$ & E69 ${ }^{0} \mid D 37 G^{-}$ & $\mathrm{NIC}$ & PYPI & PBE & 500 \\
\hline S10 & $6 \mathrm{cfw}$ & E21 $L^{-}$ & $\mathrm{NIC}$ & PYPI & PBE & 500 \\
\hline S11 & $6 \mathrm{cfw}$ & $\mathrm{E} 21_{\mathrm{L}}^{0} \mid \mathrm{H} 75 \mathrm{~L}^{+}$ & $\mathrm{NIC}$ & PYPI & PBE & 500 \\
\hline S12 & $6 \mathrm{cfw}$ & MRP Na+ & $\mathrm{NIC}$ & PYPI & PBE & 500 \\
\hline S13 & $6 \mathrm{cfw}$ & TM3D a-helical & $\mathrm{NIC}$ & PYPI & PBE & 500 \\
\hline S14 & $6 \mathrm{cfw}$ & $\mathrm{MRP} \mathrm{Na}^{+}, \mathrm{D} 37_{\mathrm{G}}{ }^{0}, \mathrm{D} 9_{\mathrm{D}}{ }^{0}$ & $\mathrm{NIC}$ & PYPI & PBE & 500 \\
\hline S15 & $6 \mathrm{cfw}$ & 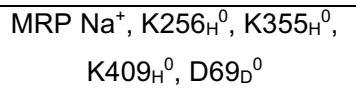 & $\mathrm{NIC}$ & PYPI & PBE & 500 \\
\hline S16 & $6 \mathrm{cfw}$ & $\mathrm{Mrp} \mathrm{Na}^{+}, \mathrm{E} 137 \mathrm{H}^{0}, \mathrm{D} 69{ }^{0}$ & NIC & PYPI & PBE & 500 \\
\hline \multicolumn{6}{|c|}{ Total: } & 8000 \\
\hline
\end{tabular}


Table S2. Non-standard pronation states used in the MD simulations. The protonation states were predicted based on Poisson-Boltzmann electrostatic calculations with Monte Carlo sampling on the in silico starting model based on PDB $6 \mathrm{cfw}$. The $\delta$-protonated imidazole is here the reference protonation state for histidines. 0: neutral; +: cationic.

\begin{tabular}{|c|c|}
\hline Residue & charge state \\
\hline Glu56D & 0 \\
\hline His61D & + \\
\hline His49E & + \\
\hline Asp62E & 0 \\
\hline Glu75E & 0 \\
\hline Asp37G & 0 \\
\hline Lys354H & 0 \\
\hline His56k & + \\
\hline Glu114k & 0 \\
\hline Glu20ı & 0 \\
\hline Glu21L & 0 \\
\hline Glu65L & 0 \\
\hline His120L & + \\
\hline Glu187L & 0 \\
\hline His192L & + \\
\hline His281 L & + \\
\hline Glu141M & 0 \\
\hline His172M & + \\
\hline Glu213m & 0 \\
\hline Glu27N & 0 \\
\hline Glu66N & 0 \\
\hline Asp96 & 0 \\
\hline Glu97N $_{N}$ & 0 \\
\hline
\end{tabular}


Table S3. In silico modeled residues in the MD setup. Missing sequences are giving in brackets, missing residues, which were modeled in silico, are colored in grey, acidic residues in red, and basic residues in blue.

\begin{tabular}{|c|c|c|c|}
\hline Subunit (first/last residue) & $\mathrm{N}$-terminal & C-terminal & Other \\
\hline$A(1-167)$ & $1(\mathrm{M})$ & $167(\mathrm{~A})$ & \\
\hline $\mathrm{B}(1-84)$ & $1(\mathrm{M})$ & $84(\mathrm{Q})$ & \\
\hline$C(3-116)$ & $1-2(\mathrm{MI})$ & 117-124 (ERGEKNES) & \\
\hline $\mathrm{D}(14-91)$ & 1-13 (MHISKKKGVRKMN) & 92-96 (EREAP) & \\
\hline$E(1-93)$ & - & 94-99 (RREENE) & \\
\hline$F(4-148)$ & 1-3 (MNE) & - & \\
\hline$G(1-114)$ & $1(\mathrm{M})$ & $115-117$ (LRG) & \\
\hline $\mathrm{H}(3-507)$ & $1-2(\mathrm{MT})$ & $508-510$ (GGG) & \\
\hline $\mathrm{I}(1-115)$ & - & $115(\mathrm{G})$ & Sidechains for $42-73$ \\
\hline$J(13-155)$ & 1-12 (MTNNSERKRLEK) & 156-167 (GEVPPEEGEENE) & \\
\hline $\mathrm{K}(4-169)$ & $1-3$ (MSK) & 170-173 (REKK) & \\
\hline$L(7-380)$ & 1-6 (MKKVEY) & - & \\
\hline$M(3-319)$ & $1-2(\mathrm{MK})$ & $320-321(\mathrm{ML})$ & \\
\hline $\mathrm{N}(1-122)$ & $1(\mathrm{M})$ & $\begin{array}{l}\text { 123-139 } \\
\text { (KKLEEANKAKAEKNASK) }\end{array}$ & \\
\hline
\end{tabular}


Table S4. Conserved polar residues in Mbh. Polar residues with a cons-surf score of at least 8 with regard to their subunit are listed. Residues with functional implications are highlighted in boldface.

\begin{tabular}{|c|c|}
\hline MbhA & $\begin{array}{l}\text { T18, Y68, N80, D82, R86, N114, S113, S115, T117, } \\
\text { T119, T122, T124, H137, E159, R164 }\end{array}$ \\
\hline MbhB & R20, D29, R30, D35, D59, Y64, Y69, T72, K77 \\
\hline MbhC & $\begin{array}{l}\text { S14, R27, D30, R34, T33, H36, T39, K40, T42, T43, } \\
\text { H75, T86, N87, H92, K96, D111 }\end{array}$ \\
\hline MbhD & S50, D64, E69, T78, T88 \\
\hline MbhE & K28, T42, Y46, N58, T61, D66, Y67, R68, D71, E75 \\
\hline MbhF & $\begin{array}{l}\text { Y26, H30, H32, T34, N40, E74, N96, S114, N122, } \\
\text { K128, K147 }\end{array}$ \\
\hline MbhG & N25, K28, H41, Q76, T81, S82, S91, T106 \\
\hline $\mathrm{MbhH}$ & $\begin{array}{l}\text { D81, Y101, S102, Y105, T132, D134, N137, E143, } \\
\text { S149, K168, Y169, K225, S226, H232, D237, H239, } \\
\text { S244, S247, S251, K256, Y261, R265, T291, Q302, } \\
\text { D304, K306, R307, Y311, S312, S313, N316, Y319, } \\
\text { H346, N349, H350, K354, T370, T386, N404, S408, } \\
\text { K409, T431, R440, T492, Y502 }\end{array}$ \\
\hline Mbhl & $\begin{array}{l}\text { D6, Y9, T35, R36, K43, S47, E49, D50, E59, H60, T66, } \\
\text { N69, H89, T90, D95, Y96, Y99, T103 }\end{array}$ \\
\hline MbhJ & $\begin{array}{l}\text { S24, N31, C35, N36, C38, E41, D51, R54, S62, R64, } \\
\text { H65, D67, T72, Q78, Y87, Q89, T90, C102, S107, } \\
\text { D126, C132 }\end{array}$ \\
\hline MbhK & $\begin{array}{l}\text { K26, H56, D64, N73, H74, K95, D102, T103, D105, } \\
\text { S110, E114, R115, E116, E119, D130, R132, D139, } \\
\text { Y145, R148, H162, Y166 }\end{array}$ \\
\hline MbhL & $\begin{array}{l}\text { H16, E21, E30, R46, Q49, E65, R66, C68, C71, S72, } \\
\text { H75, E84, R94, R99, E104, E106, R107, H109, S110, } \\
\text { H111, H120, T126, H129, R135, E136, E143, N148, } \\
\text { R149, D163, R199, R222, S224, D229, R231, Y239, } \\
\text { D260, R264, R268, E271, S275, E317, R320, E322, } \\
\text { H325, R338, K340, R342, E343, T345, N348, D363, } \\
\text { S370, D372, C374, S376, C377, T378, D379, R380, }\end{array}$ \\
\hline MbhM & $\begin{array}{l}\text { S21, D28, R29, K30, Q36, R38, Q45, D49, K52, K56, } \\
\text { Y85, D99, Y105, S121, S123, R132, S139, R140, } \\
\text { E141, E199, E201, N206, E209, E211, Q212, E213, } \\
\text { E221, Y222, S223, Y226, K237, N265, K272, T282, } \\
\text { R285, R290, Q295 }\end{array}$ \\
\hline MbhN & T20, R36, C46, C49, C52, C56, C77, C80, C83, C87, \\
\hline
\end{tabular}


Table S5. Benchmarking different density functionals for DFT models of the NiFe site in Mbh. Electronic energies are evaluated in the Ni"/Fe" $(S=1)$ state with def2-TZVP basis sets and $\varepsilon=4$. The energies are reported in $\left(\mathrm{kcal} \mathrm{mol}^{-1}\right)$. GluH - Glu21 $\mathrm{L}$ protonated; $\left[\mathrm{H}_{3} \mathrm{O}^{+}\right]^{\ddagger}$ transition state for $\mathrm{pT}$ between Glu21 L and Cys374L; CysH - Cys374L protonated; $\mathrm{CysH}_{\text {flip }}$ - Cys374L protonated with proton flipped towards the $\mathrm{H}^{-} ; \quad[\mathrm{H} . . . \mathrm{H}]^{\ddagger}$ transition state for $\mathrm{H}_{2}$ formation; $\mathrm{H}_{2}-\mathrm{H}_{2}$ product state. The amount of $\mathrm{HF}$ exchange (xc) or short-range (sr)/long-range (Ir) exchange for the range-separated functionals are reported in parentheses.

\begin{tabular}{|c|c|c|c|c|c|c|}
\hline State & $\begin{array}{c}\text { B3LYP-D3 } \\
(20 \% \text { xc })\end{array}$ & $\begin{array}{c}\text { B3LYP } \\
(20 \% \text { xc) }\end{array}$ & $\begin{array}{c}\text { B3LYP*-D3 } \\
(15 \% \text { xc })\end{array}$ & $\begin{array}{c}\text { CAM- } \\
\text { B3LYP-D3 } \\
\text { (19\% sr xc, } \\
65 \% \text { Ir xc) }\end{array}$ & $\begin{array}{c}\text { CAMh- } \\
\text { B3LYP-D3 } \\
\text { (19\% sr xc, } \\
50 \% \text { Ir xc) }\end{array}$ & $\begin{array}{c}\omega \text { B } 97 X \mathbf{X} \\
(22 \% \text { sr } x c, \\
100 \% \text { Ir } \times c)\end{array}$ \\
\hline GluH & 0.0 & 0.0 & 0.0 & 0.0 & 0.0 & 0.0 \\
\hline$\left[\mathrm{H}_{3} \mathrm{O}^{+}\right]^{\ddagger}$ & 9.0 & 9.2 & 8.2 & 9.4 & 9.4 & 10.5 \\
\hline CysH & 0.6 & -1.0 & 0.7 & 1.0 & 0.4 & 1.6 \\
\hline $\mathrm{CysH}_{\text {flip }}$ & 0.0 & 0.0 & 0.0 & 0.0 & 0.0 & 0.0 \\
\hline$[\mathrm{H} \ldots \mathrm{H}] \neq$ & 24.8 & 27.9 & 23.5 & 27.8 & 27.4 & 27.9 \\
\hline $\mathrm{H}_{2}$ & 7.8 & 12.7 & 7.4 & 9.3 & 9.4 & 10.5 \\
\hline
\end{tabular}




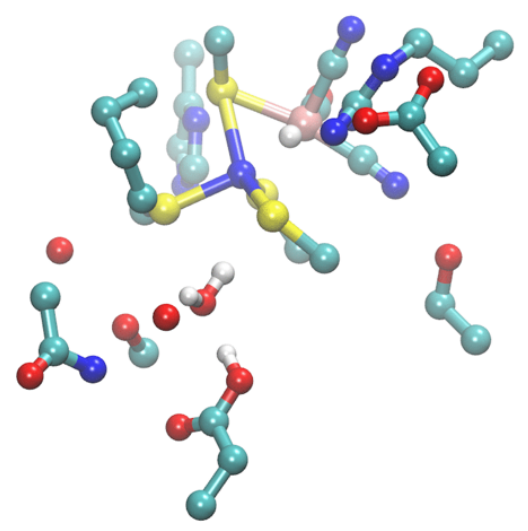

Movie S1. Movie of water-mediated proton transfer from Glu21 $\mathrm{L}$ via Cys374 $\mathrm{L}$ to $\mathrm{H}$. The animation is based on reaction pathway optimization for $\mathrm{PT}$ from Glu21 L to Cys374L and Cys374L to $\mathrm{H}^{-}$(see main text and Methods). 


\section{SI References}

1. Baker, N. A.; Sept D.; Joseph, S.; Holst, M. J.; McCammon, J. A. Electrostatics of nanosystems: Application to microtubules and the ribosome. Proc. Natl. Acad. Sci. USA 2001 98, 10037-10041. 2. B. R. Brooks, et al. CHARMM: The biomolecular simulation program, J. Comput. Chem. 2009 30, 1545-1614.

3. Kieseritzky, G.; Knapp, E. W. Improved $p K_{a}$ prediction: Combining empirical and semimicroscopic methods. J. Comput. Chem. 2008, 29 (15), 2575-2581.

4. Rabenstein, B.; Ullmann, G. M.; Knapp, E-W; Calculation of protonation patterns in proteins with structural relaxation and molecular ensembles - application to the photosynthetic reaction center. Eur. Biophys. J.1998 27, 626-637.

5. Plessow, P. Reaction path optimization without NEB springs or interpolation algorithms, J. Chem. Theory Comput. 2013 9, 1305-1310.

6. Becke, A. D. Density-functional thermochemistry. III. The role of exact exchange. J. Chem. Phys. 1993, 98, 5648-5652.

7. Reiher, M., Salomon, O.; Hess, B. A. Reparameterization of hybrid functionals based on energy differences of states of different multiplicity Theor. Chem. Acc. 2001, 107, 48-55.

8. Yanai, T.; Tew, D. P.; Handy, N. C. A new hybrid exchange-correlation functional using the Coulombattenuating method (CAM-B3LYP). Chem. Phys. Lett. 2004 393, 51-57.

9. Shao, Y.; Mei, Y.; Sundholm, D.; Kaila, V. R. I. K. Benchmarking the Performance of Time-Dependent Density Functional Theory Methods on Biochromophores J. Chem. Theory Comput. 2020 16, 1, 587600.

10. Da Chai, J.; Head-Gordon, M.; Long-range corrected hybrid density functionals with damped atomatom dispersion corrections. Phys. Chem. Chem. Phys. 2008, 10, 6615-6620.

11. Song, W.; Corey, R. A.; Ansell, T. B.; Cassidy, C. K.; Horrell, M. R.; Duncan, A. L.; Stansfeld, P. J.; Sansom, M. S. P.; PyLipID: A Python package for analysis of protein-lipid interactions from MD simulations. BioRxiv 2021, https://doi.org/10.1101/2021.07.14.452312

12. D. N. Grba, J. Hirst. Mitochondrial complex I structure reveals ordered water molecules for catalysis and proton translocation. Nat. Struct. Mol. Biol. 2020, 27, 892-900.

13. A. Jussupow, A. DI Luca, V.R.I. Kaila. How cardiolipin modulates the dynamics of respiratory complex I. Sci. Adv. 2019, 5, 31850.

14. Wang, P.; Shi, R.; Su, Y.; Tang, L.; Huang, X.; Zhao, J. Hydrated Sodium lon Clusters [Na+(H2O)n $(n=1-6)]$ : An Ab Initio Study on Structures and Non-Covalent Interaction. Front. Chem. 2019, 7, 624. 